\title{
Knockdown of Annexin A1 induces apoptosis, causing G2/M arrest and facilitating phagocytosis activity in human leukemia cell lines
}

MASYITAH HASAN ${ }^{1}$

ENDANG KUMOLOSASI ${ }^{1, *}$

IBRAHIM JANTAN $^{2}$

MALINA JASAMAI ${ }^{1}$

NORLAILI NAZARUDIN ${ }^{1}$

${ }^{1}$ Drug and Herbal Research Centre

Faculty of Pharmacy, Universiti

Kebangsaan Malaysia, Kuala Lumpur

Malaysia

${ }^{2}$ Institute of Systems Biology, Universiti Kebangsaan Malaysia, 43600 UKM

Bangi, Selangor, Malaysia

\begin{abstract}
Annexin A1 (ANXA1) is an endogenous protein involved in the control of proliferation, cell cycle, phagocytosis, and apoptosis in several types of cancer. To investigate the effects of ANXA1 knockdown in leukemia cells, transfection with specific ANXA1 siRNA was performed. Cell cycle and apoptosis were analyzed using flow cytometry and a mechanism involving caspases and Bcl-2 was quantified using Western blotting. Phagocytosis activity was evaluated using hematoxylin \& eosin staining. The ANXA1 expression was significantly downregulated after the knockdown and apoptosis was induced in tested cells. The expression of caspase-9 and -3 increased in U937 and Jurkat cells respectively. Bcl-2 expression was downregulated in K562 and Jurkat cells while upregulated in U937. The number of leukemic cells arrested at the G2/M phase and the phagocytosis index were significantly increased in transfected cells. This suggests that ANXA1 knockdown might be a potential approach in the therapeutic strategy for leukemia.
\end{abstract}

Keywords: Annexin A1, siRNA transfection, apoptosis, cell cycle, phagocytosis, leukemia

Annexin A1 is a $37 \mathrm{kDa}$ protein with calcium and phospholipid-binding properties involved in various physiological processes including inflammation, cell proliferation, migration, differentiation, phagocytosis and apoptosis in multiple types of cancer. This cascade of signaling pathways was activated through the association of ANXA1 with formyl peptide receptors (FPRs) (1). Recently, a study reported that ANXA1 level was significantly higher in leukemia cells (Jurkat, K562 and U937) compared to peripheral blood mononuclear cells (PBMC) (2). The role of Annexin A1 in the mechanisms of the mentioned processes is not completely clear, especially in leukemia.

Solito et al. reported that the treatment of human neutrophils with exogenous ANXA1 stimulates apoptosis (3). A previous study showed that the overexpression of ANXA1 induced apoptosis with caspase- 3 activation in macrophages and bronchoalveolar cells (4, 5). Apoptosis is a process regulated by certain caspase family and B-cell lymphoma 2

\footnotetext{
*Correspondence; e-mail: e_kumolosasi@ukm.edu.my; e_kumolosasi@yahoo.co.id
} 
(Bcl-2) family (6). Caspases are produced in an inactive form called zymogens and are cleaved into active form during the apoptotic event. The activation of caspases, a family of cysteine-protease proteins and one of the hallmarks of apoptosis, can be categorized into initiator and effector caspases. The activation of initiator caspases (such as caspase- 9 and -10) through the cleavage of aspartate (Asp) residue triggers the execution of effector caspases (such as caspase-3 and -7). Extrinsic and intrinsic pathways, involving death ligand-receptor and mitochondria respectively, are two major apoptosis pathways. The induction of apoptosis over the intrinsic pathway is associated with the release of cytochrome c from mitochondria that activates the initiator caspase, pro-caspase- 9 ( $47 \mathrm{kDa})$ and is activated by cleavage into the active form, caspase- 9 ( $35 \mathrm{kDa})$. It then cleaves procaspase- 3 (35 kDa) into $19 \mathrm{kDa}$ and $17 \mathrm{kDa}$ subunits. Meanwhile, the extrinsic pathway involves the activation of caspase- 8 or caspase- 10 through the death ligand-receptor and the activation of initiator caspases subsequently activates the effector caspase, caspase-3 (7). The common anti-cancer mechanism in cancer therapy is through induction of apoptosis (8). Bcl-2 is classified as an anti-apoptotic protein that is usually located in the outer membrane of mitochondria. The function of this protein is to protect mitochondrial membrane potential and prevent the release of cytochrome $\mathrm{c}$ or other pro-apoptotic proteins from the mitochondria. Localization of $\mathrm{Bcl}-2$ in the inner membrane of mitochondria protects mitochondria from apoptosis and subsequently, resistance toward chemotherapeutic drugs as most of it is targeting mitochondria (9).

Besides, ANXA1 is able to alter cell cycle progression in few cancer cells including leukemia cells (10). A previous study reported that ANXA1 is involved in phagocytosis activity since knockdown of ANXA1 in RAW264.7 macrophages reduced the phagocytic activity (11). Therefore, the objective of the present study is to explore the effect of ANXA1 knockdown on apoptosis, cell cycle arrest and phagocytosis activities in different types of leukemia. Thus, different leukemic cell lines (U937 cells, Jurkat cells, THP-1 and K562) were used as model cell lines for studying different types of leukemia.

\section{EXPERIMENTAL}

\section{Cell cultures}

The acute myeloid leukemia, U937 cells, acute lymphoblastic leukemia, Jurkat cells and acute monocytic leukemia, THP-1 cells were cultured in RPMI 1640 medium. Meanwhile, chronic myeloid leukemia, K562 cells, were grown in Iscove's Modified Dulbecco's medium, IMDM. The cultured media were enriched with $10 \%$ of fetal bovine serum (FBS), $1 \%$ of penicillin-streptomycin. The cells were obtained from American Type Culture Collection (Manassas, VA, USA).

\section{Transfection with small interfering RNA (siRNA) of ANXA1}

The Jurkat, U937 and $\mathrm{K} 562$ cells $\left(1.5 \times 10^{5}\right.$ cell $\left.\mathrm{mL}^{-1}\right)$ were transiently transfected with 40, 80 and $100 \mathrm{nmol} \mathrm{L}^{-1}$ respectively of ANXA1 small interfering RNA (siRNA), (Silencer ${ }^{\circledR}$ Select Validated siRNA; siRNA ID s1381 Ambion, TX, USA) using the INTERFERin transfection reagent (Polyplus-transfection Inc., France). Each of the experiments was done three times. Positive control of siRNA, fluorescein isothiocyanate, FITC conjugated fluorescent 
oligonucleotide (Block-it, Invitrogen) were analyzed using fluorescence microscopy to evaluate transfection efficiency $24 \mathrm{~h}$ post-transfection. The ANXA1 expression was quantified using Western blotting $72 \mathrm{~h}$ post-transfection.

\section{Apoptosis assay}

Post transfection, the ANXA1 knockdown cells were stained with Annexin V/propidium iodide to quantify the percentage of apoptotic cells. The early marker of apoptosis is the externalization of phosphatidylserine (PtdSer) from the inner to the outer membrane. Annexin V was used due to its high affinity towards PtdSer, thus binding to PtdSer, exposed on the outer membrane, served as a probe for detection. The cells were washed with cold PBS and incubated with a staining solution containing propidium iodide, FITC-labeled Annexin $\mathrm{V}$ and binding buffer for $10 \mathrm{~min}$. The percentage of viable, apoptotic and necrotic cells was assessed by FACSCanto II flow cytometer (Becton Dickinson, USA).

\section{Western blotting}

Western blotting was conducted to determine the protein expression level of ANXA1 and apoptosis proteins post knockdown. All of the proteins were extracted with RIPA lysis buffer (MERCK) and quantified using Bradford protein assay (Bio-Rad, USA). The same amount of protein was separated using $10 \%$ SDS-polyacrylamide gel electrophoresis (SDSPAGE), transferred to polyvinylidene fluoride (PVDF) membranes (Merck, Germany) and blocked with $5 \%$ milk in Tris-buffered Saline, $0.1 \%$ Tween 20 (TBST) for $1 \mathrm{~h}$ at room temperature. The membranes were incubated overnight with antibodies of ANXA1, caspase-1, caspase-3, caspase-9, Bcl-2, and $\beta$-actin (1:1000) as a loading control (Cell Signaling Technology, USA). Then, membranes were washed, incubated for $1 \mathrm{~h}$ with secondary anti-rabbit horseradish peroxide (HRP)-conjugated antibody (1:5000) (Cell Signaling Technology) and visualized using the enhanced chemiluminescence (ECL) detection kit on ChemiDoc MP System (Bio-Rad, USA).

\section{Cell cycle assay}

Cells were washed twice with cold phosphate-buffered saline (PBS) and fixed in cold $70 \%$ ethanol. Post $2 \mathrm{~h}$ of fixation process, cells were rinsed with PBS, stained for $30 \mathrm{~min}$ with propidium iodide buffer containing $0.2 \mathrm{mg} \mathrm{mL}^{-1}$ of propidium iodide, $0.2 \mathrm{mg} \mathrm{mL}^{-1}$ of RNase A and PBS in the darkroom. The cell cycle phases were conducted using a FACSCanto II flow cytometer and the result was analyzed using ModFit LT software (Becton Dickinson, USA). Propidium iodide is a DNA-intercalating agent used to analyze DNA content in the cell cycle.

\section{Phagocytosis assay}

THP-1 cells were differentiated into macrophages for $48 \mathrm{~h}$ after treated with $20 \mathrm{ng}$ $\mathrm{mL}^{-1}$ of phorbol 12-myristate 13-acetate (PMA). The non-transfected and transfected cells were co-cultured with macrophages for $3 \mathrm{~h}$ and stained with hematoxylin and eosin. The percentage of phagocytosis and phagocytosis index were calculated using the equations (1) and (2): 


$$
\begin{gathered}
\text { Percentage of phagocytosis }(\%)=\frac{b}{a} \times 100 \\
\text { Phagocytosis Index (PI) }=\frac{c b}{a}
\end{gathered}
$$

where $a$ is the number of macrophages, 200, $b$ is the number of macrophages that engulfed apoptotic cells and $c$ is the number of apoptotic cells engulfed by macrophages.

\section{Statistical analysis}

All results are presented as mean and standard error mean, SEM and were statistically analyzed using $t$-test by GraphPad Prism 5 Software (CA, USA). The targeted protein bands' intensities detected by Western blotting were normalized to $\beta$-actin. Differences were considered significant when $p$ values less than 0.05 .

\section{RESULTS AND DISCUSSION}

\section{Knockdown of ANXA1 expression using siRNA}

In order to determine the ANXA1 effects on cell apoptosis, cell cycle arrest and phagocytosis activities in leukemia cells, knockdown using ANXA1 siRNA transfection was carried out. Firstly, the transfection efficiency was determined using BLOCK-iT Fluorescent control siRNA. After $24 \mathrm{~h}$ of transfection, the knockdown cells were observed using a fluorescent microscope. Fig. 1a shows the image of transfected leukemia cells. The red fluorescence cells represent successfully transfected cells with BLOCK-IT siRNA. Cells that do not emit any fluorescent light were considered non-transfected cells. The ANXA1 expression of transfected cells was detected using Western blot. The results presented in the figure below showed significant downregulation of ANXA1 expression in the ANXA1 transfected cell, compared to the negative control, non-transfected cell post-transfection. The ANXA1 expression of transfected Jurkat, K562 and U937 was significantly decreased by 70,69 and $63 \%$ as compared to non-transfected cells, respectively. These results demonstrated that ANXA1 expression successfully decreased in leukemia cells. Thus, the ANXA1 transfected cells were assessed for apoptosis, cell cycle, and phagocytosis assay.

\section{Knockdown of ANXA1 with siRNA induces apoptosis and necrosis}

To determine the effect of ANXA1 knockdown in affecting apoptotic cells, cells were measured using flow cytometry. The percentage of viable, apoptotic, and necrotic cells was quantified using flow cytometry. The distribution of apoptotic cells was determined as those in early apoptosis (Quadrant 4 (Q4), lower right quadrant), late apoptosis (Quadrant 2 (Q2), upper right quadrant) and necrotic cells in Quadrant 1 (Q1), upper left quadrant. Fig. 2 showed that the percentage of viable cells in quadrant 3 (Q3) decreased significantly in K562 cells. The relative percentage change of apoptotic cells was significantly increased in the transfected Jurkat (138.9\%), K562 (89.7\%) cells with $p<0.05$ for both cell lines and U937 (49.4\%) cells with $p<0.01$ in comparison to its respective non-transfected cells. 
a)

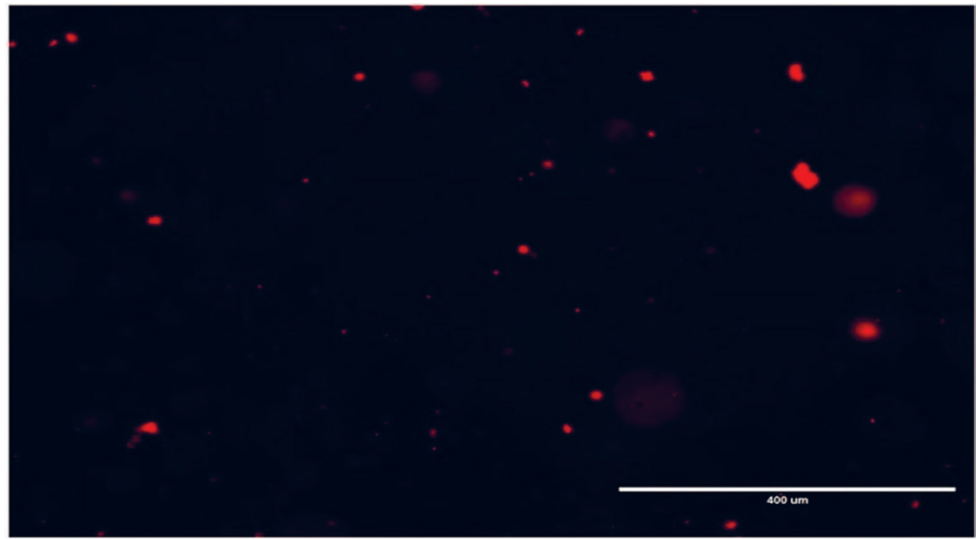

b)
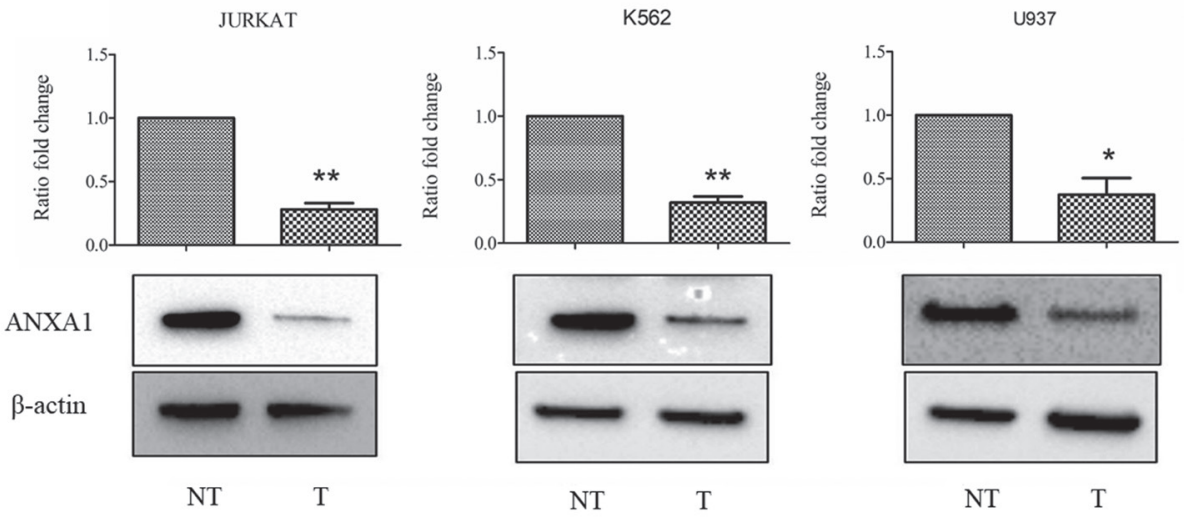

Fig. 1. a) The transfected cells (coloured red) were observed under a fluorescence microscope (100× magnification). b) The quantification of ANXA1 protein expression using Western blotting analysis showed that ANXA1 protein expression was downregulated in the transfected cell (T) compared to the non-transfected cell (NT). $\beta$-actin was used as a loading control. Data are presented as mean \pm SEM; $n=3,\left({ }^{*} p<0.05,{ }^{* *} p<0.01\right)$. Full-length blots are presented in Supplementary Data Fig. 1.

Necrosis is marked by nonspecific swelling of cells and disruption on cell membrane integrity. This will cause externalization of cell content to the outer environment that can cause inflammation. ANXA1 knockdown significantly increased the relative percentage change of necrotic cells (617.9\%) with $p<0.05$ in K562 cells.

In this study, suppression of ANXA1 in Jurkat, K562 and U937 cells caused significant apoptosis. These indicated that suppression of ANXA1 expression induced apoptosis in Jurkat, K562, and U937 cells. This finding was supported by Chen et al. who revealed that ANXA1 knockdown inhibits cell proliferation and induces apoptosis in human T cell leukemia virus type I, HTLV-I-transformed T cell (12). The previous research findings have shown that ANXA1 is involved in the apoptosis process; some studies suggested ANXA1 as a pro-apoptotic protein, whereas others demonstrated that ANXA1 acts as an anti-apoptotic protein in certain cancer cells. Wu et al. reported that a high level of ANXA1 inhibits 
a)
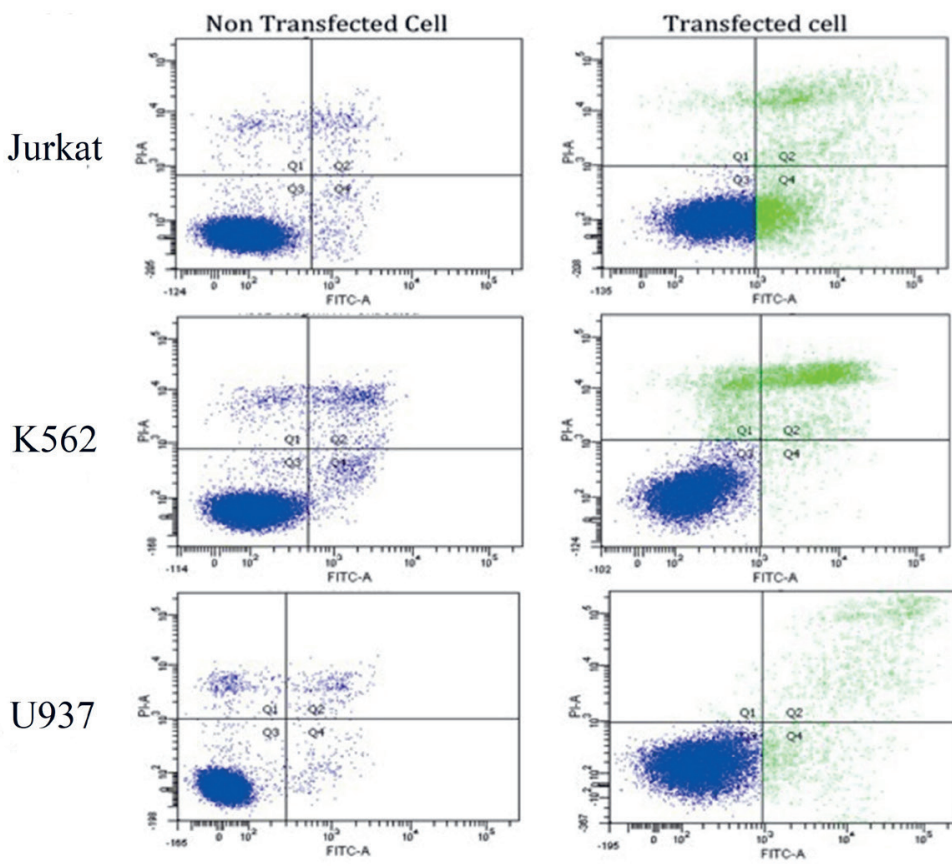

b)

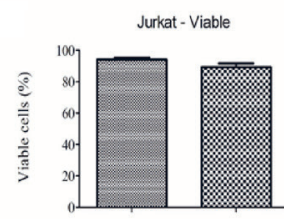

Jurkat - Apoptosis

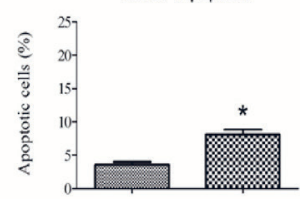

Jurkat - Necrosis

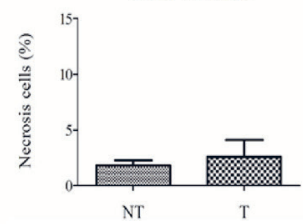

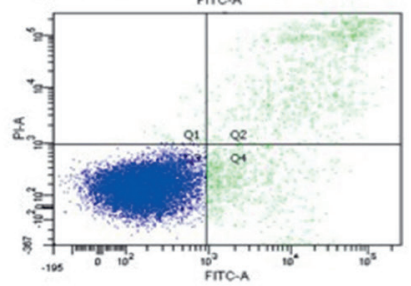

K562 - Viable

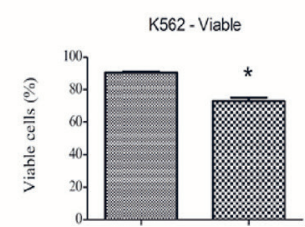

K562 - Apoptosis

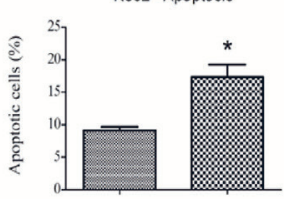

K562 - Necrosis

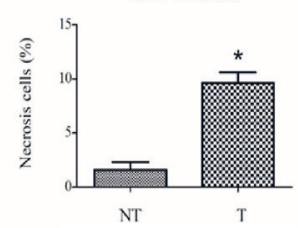

U937 - Viable

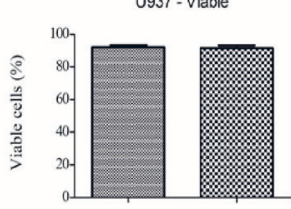

U937 - Apoptosis

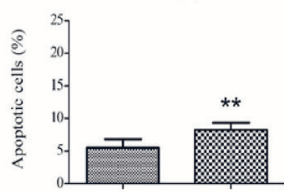

U937 - Necrosis

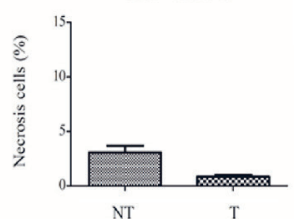

Fig. 2. a) A dot plot of the cell distribution percentage after ANXA1 knockdown. Q1: Necrotic cells, Q2: Late apoptotic cells, Q3: Viable cells, Q4: Early apoptotic cells. b) The effects of ANXA1 knockdown on cell viability, apoptosis and necrosis in Jurkat, K562 and U937 cells. T: transfected cells, NT: non-transfected cells. The value expressed as mean \pm standard error mean (SEM); $n=3,{ }^{*}(p<0.05)$ and ${ }^{* *}(p<0.01)$ when compared with non-transfected cells. 
M. Hasan et al:: Knockdown of Annexin A1 induces apoptosis, causing G2/M arrest and facilitating phagocytosis activity in human leukemia cell lines, Acta Pharm. 72 (2022) 109-122.

apoptosis and might protect them against immune surveillance in human leukemic cells (13). Moreover, Jia and colleagues found that high ANXA1 expression was reported in multiple myeloma cells, NCI-H929 and RPMI8226 and after silencing of ANXA1 in both cells, apoptosis was induced (14). The suppression of ANXA1 protein might cause disruption in the normal condition of leukemic cells and eventually caused cell death. This indicated that ANXA1 might serve as an oncogene protein in these types of leukemia cells. Even though the percentage of necrotic cells decreased non significantly in transfected U937 cells, this is probably because of the phagocytic ability possessed by this cell line as monocytes are classified as professional phagocytes (15). To further confirm the involvement of ANXA1 in apoptosis, the molecular mechanism study was conducted.

\section{Mechanism of apoptosis in cells transfected with siRNA for ANXA1}

To evaluate the molecular mechanism of apoptosis through the activation of caspases, Western blotting was performed. Knockdown of ANXA1 significantly induced cell death and this is probably related to the increase of pro-caspase- 3 and pro-caspase- 9 expression. As shown in Fig. 3, transfected cells cause an increase of the initiator caspase, pro-caspase-9 expression in U937 but a decrease in K562 cells. Meanwhile, the effector caspase, pro-caspase-3 expression was increased in Jurkat and decreased in U937 cells. However, no caspase cleavage was observed. Bcl-2 expression showed upregulation in U937 but downregulated in K562 cells. Caspase- 1 is an enzyme involved in the activation of the proinflammatory cytokine, however, western blotting showed an incresed procaspase-1 level in all cells after ANXA1 knockdown.

The Western blotting technique was performed to study the molecular mechanism involved in the induction of apoptosis after ANXA1 knockdown. Based on this study, the expression of pro-caspase- 3 and pro-caspase- 9 showed a slight increase in certain cells however no cleavage of these caspases was observed. Feng et al. demonstrated that silencing of ANXA1 reduced apoptosis by upregulating Bcl-2 and procaspase-3 levels while downregulating the expression of cleaved caspase- 3 in trophoblasts from preeclampsia rat's placenta tissue (16). The cleavage of caspase- 9 and caspase- 3 in this study was not observed probably due to approximately $10 \mathrm{~min}$ of caspase activation when there was a trigger of cell destruction. The execution of apoptosis begins with permeabilization of the mitochondrial membrane, the release of pro-apoptotic proteins into the cytosol then activates caspases (17). Induction of apoptosis in U937 cells may involve an intrinsic pathway with an increased level of pro-caspase- 9 and decreased level of pro-caspase-3 expression after ANXA1 knockdown. Meanwhile, knockdown of ANXA1 in K562 cells caused a decrease in procaspase- 9 and an increase in procaspase- 3 expression that induced apoptosis through the extrinsic pathway. Likewise, in Jurkat cells, knockdown of ANXA1 increased procaspase-3, caused no change in pro-caspase- 9 expression and induced apoptosis through the extrinsic pathway that may be involved in the activation of caspase- 8 or caspase- 10 .

This study proves that Bcl-2 expression in K562 cells showed a slight reduction compared to non-transfected cells. The decrease of Bcl-2 expression with no disruption of mitochondrial membrane potential (MMP) might be caused by a significant decrease of pro-caspase-9 expression in K562 cells. On the contrary, in U937 cells apoptosis occurs despite an increase in Bcl-2 expression levels. This may due to the increase of pro-caspase- 9 expression because there is a disruption of MMP where Bcl-2 takes action in protecting MMP and preventing apoptosis. The previous study reported that $\mathrm{Bcl}-2$ expression is overexpressed 


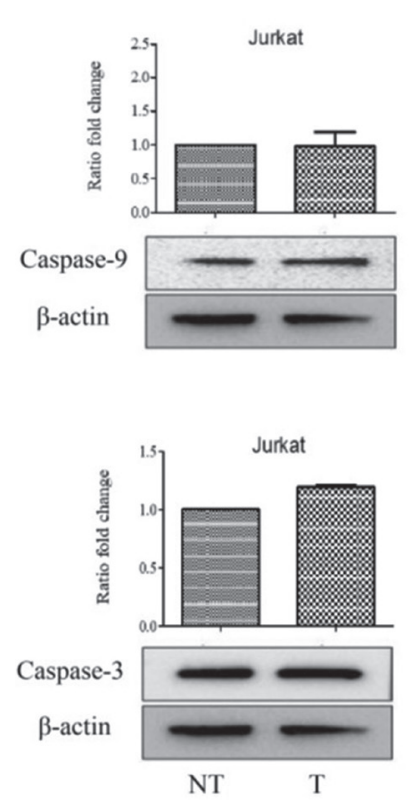

Caspase-9
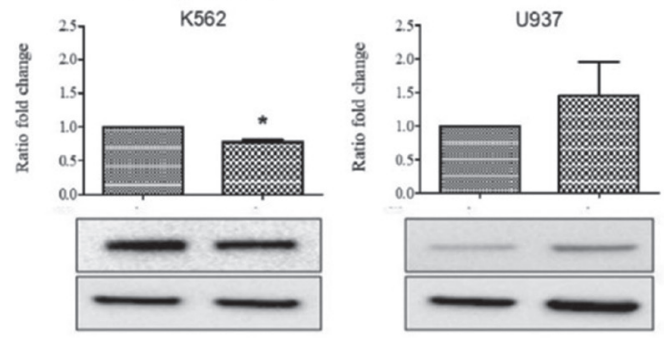

\section{Caspase-3}

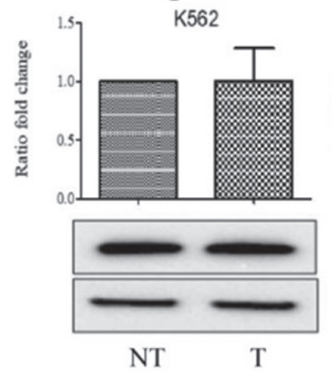

Bcl-2
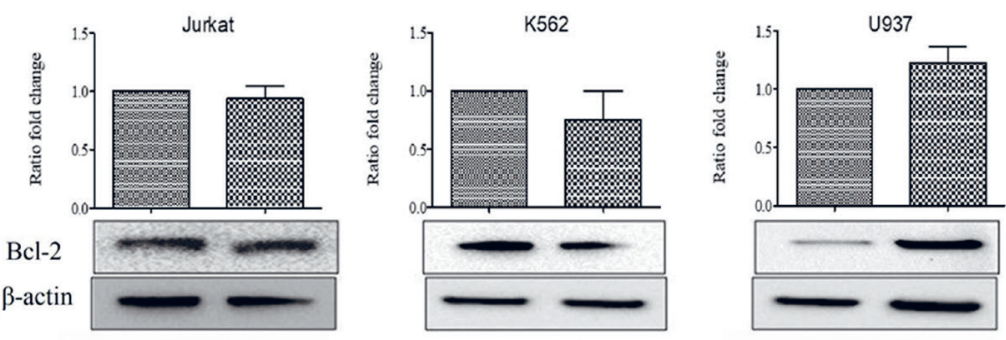

Caspase-1
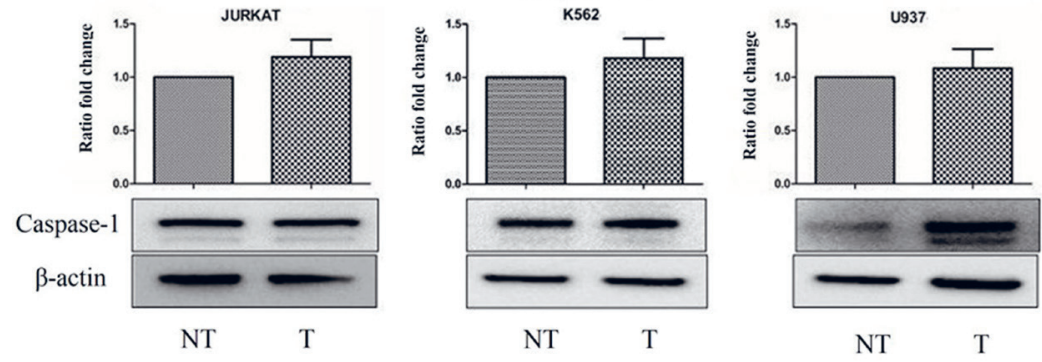

Fig. 3. Expression of caspases $-9,-3,-1$ and Bcl-2 proteins in non-transfected (NT) and transfected (T) cells were quantified using Western blotting. The value are expressed as mean \pm standard error mean (SEM), $n=3,^{*}(p<0.05)$ when compared with non-transfected cells. Full-length blots are presented in Supplementary Data Fig. 3. 

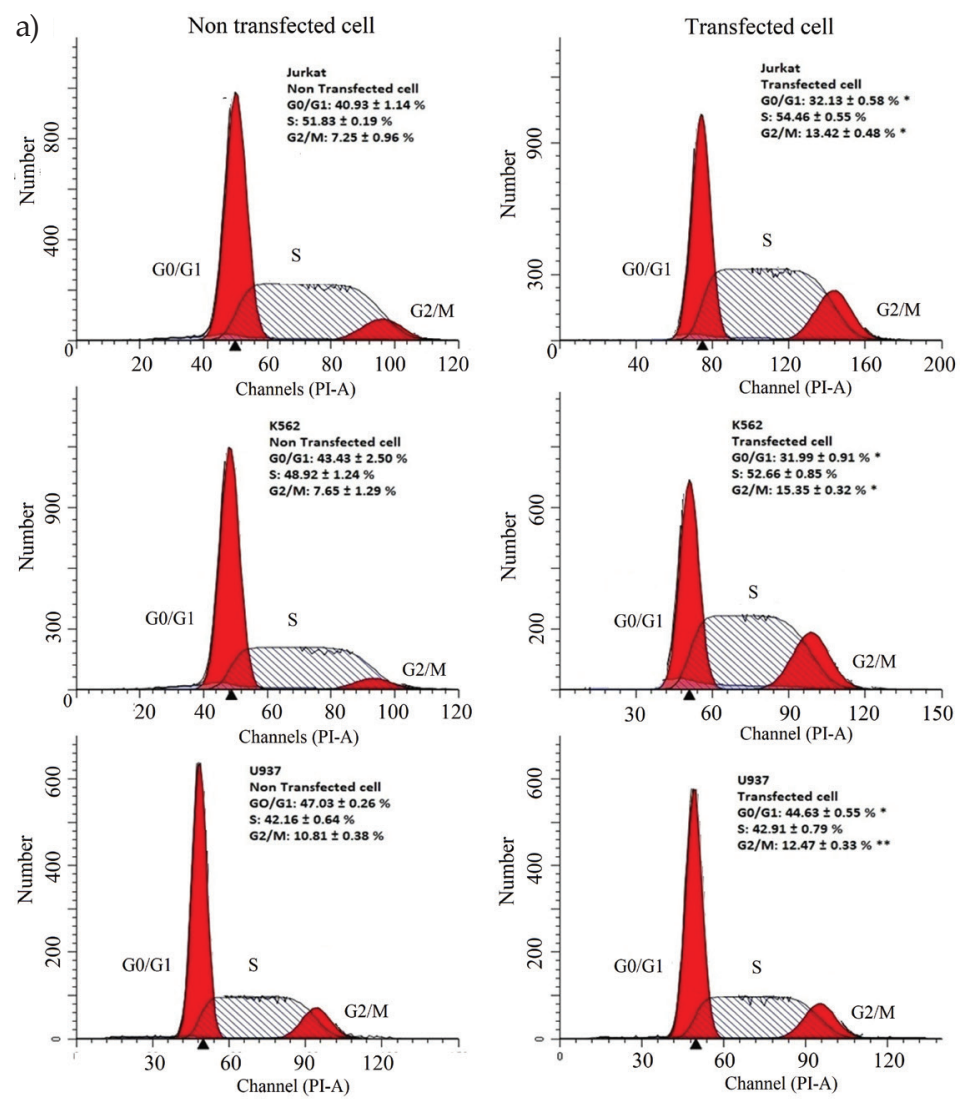

b)
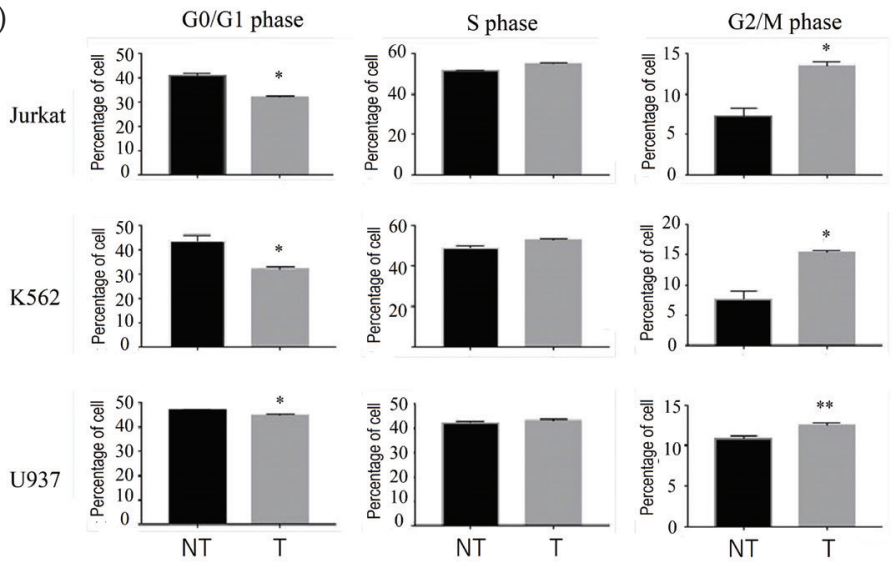

Fig. 4. a) Flow cytometry analysis and b) Bar chart of cell cycle distribution after ANXA1 knockdown in transfected (T) Jurkat, K562 and U937 cells compared to non-transfected cells (NT). Data are presented as mean \pm SEM; $n=3,{ }^{*} p<0.05,{ }^{* *} p<0.01$ compared to non-transfected cell. 
in leukemic cancer such as acute myeloid leukemia (9). This suggests why leukemic cells are resistant to chemotherapeutic agents. Prior activation of caspase-9, apoptotic stimuli causes the activation of pro-apoptotic protein such as Bax or Bak and release of apoptosis promoting factors such as cytochrome c, Smac/DIABLO and apoptosis-inducing factor (AIF). This finding was supported by the previous study done by Moore and Letai which showed that the overexpression of Bcl-2 did not prevent the cells from undergoing apoptosis (18).

This finding showed an increase of pro-caspase-1 expression after ANXA1 knock -down in all leukemic cells, however, no cleavage of caspase-1 was observed. Knockdown of ANXA1 halted the ability of ANXA1 to act as an anti-inflammatory protein which increased pro-caspase-1 expression due to inflammation. Caspase-1 activation cleaved proinflammatory cytokines (pro-IL-1 $\beta$ and pro-IL-18) into active forms and activated the effector caspase, caspase-7 as well (19). Caspase-1 involved in pro-inflammatory cytokines activation during inflammation and ANXA1 was first discovered as an endogenous antiinflammatory glucocorticoid-inducible protein by inhibiting cytosolic phospholipase $\mathrm{A}_{2}$ (cPLA_2), cyclooxygenase-2 (COX2) and consequently suppressing prostaglandin and leukotriene production (20). Activation of caspase-1 in cultured mouse cortical neurons triggers $\mathrm{BH} 3$-interacting domain (Bid) cleavage into truncated $\mathrm{Bid}, \mathrm{tBid}$ and activates a mitochondrial pathway that induces apoptosis (21).

\section{Knockdown of ANXA1 induces cell cycle arrest at the G2/M phase}

Cell cycle arrest is an important process for repairing damaged DNA before entering into another phase. To determine the effect of ANXA1 suppression in affecting cell cycle progression, staining with propidium iodide and analysis using flow cytometry was conducted. After transfection, ANXA1 knockdown resulted in a significant reduction in G0/ G1 phase and a significant increase in the G2/M phase at transfected cells in comparison to non-transfected cells. Fig. 4 showed that knockdown of ANXA1 caused the relative percentage change of the G2/M phase to increase significantly by $85.1 \%$ for Jurkat, $100.7 \%$ for K562 with $p<0.05$ for both cell lines and $15.4 \%$ for U937 with $p<0.01$ compared to its non-transfected cells. This can be observed as a significant decrease in the relative percentage change of the G0/G1 phase with $p<0.05$ for 3 cell types tested; a increase by $21.5 \%$ for Jurkat cell, $26.3 \%$ for K562 cell and $5.1 \%$ for U937 cell compared to respective non-transfected cells. Therefore, this causes the cell cycle arrest at the G2/M phase in Jurkat, K562, and U937 cells.

Results obtained showed that after ANXA1 knockdown, cell cycle progression was arrested at the G2/M phase with a decrease in the cell population at the G0/G1 phase in all cells. This suggests that without ANXA1, there is DNA damage causing cell cycle arrest to occur at the G2/M phase. This finding was supported by the previous study done by Liu et al. who reported that ANXA1 silencing caused cell cycle arrest at the G0/G1 phase in MIA PaCa-2 and BxPC3, human pancreatic cells (22). Besides, Li and colleagues showed that an increase in ANXA1 caused arrest at the G0/G1 phase and supported this finding which showed ANXA1 knockdown caused the G2/M arrest (23).

\section{Effect of ANXA1 knockdown on phagocytosis activity}

To determine the effect of ANXA1 knockdown on phagocytosis activity, hematoxylineosin staining was conducted. The percentage of phagocytosis was determined as the number of macrophages that actively phagocytose. Meanwhile, the phagocytosis index (PI) was defined as the number of apoptotic cells engulfed by each phagocytically active 
a)

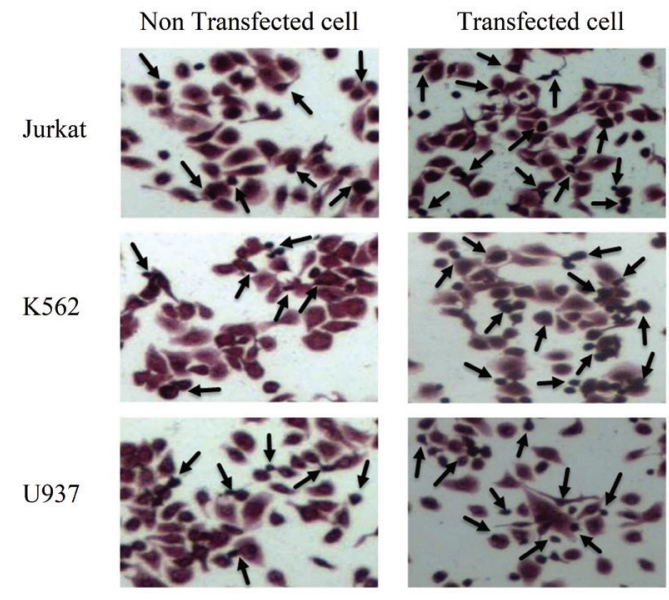

b)
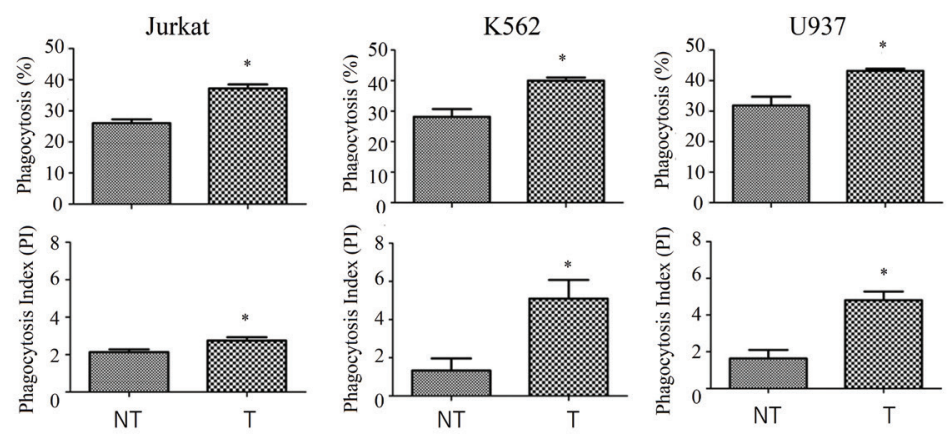

Fig. 5. a) Haematoxylin-eosin staining of cells with arrows indicating apoptotic cells engulfed by macrophages. (100× magnification). b) The percentage of phagocytosis and phagocytosis index (PI) are significantly higher in transfected cells (T) compared to non-transfected cells (NT). Data are presented as mean \pm SEM; $n=3,{ }^{*} p<0.05$ compared to non-transfected cell.

macrophage. Results shown in Fig. 5 describe that the knockdown of ANXA1 significantly increased the relative percentage change of phagocytosis in Jurkat $(42.9 \%, p<0.05), \mathrm{K} 562$ $(42.0 \%, p<0.05)$ and U937 $(35.6 \%, p<0.05)$ compared to its non-transfected cell. Phagocytosis index (PI) of ANXA1 siRNA Jurkat $(2.75 \pm 0.17, p<0.05), \mathrm{K} 562(5.43 \pm 0.88, p<0.05)$ and U937 (4.81 $\pm 0.48, p<0.05)$ cells is significantly increased compared to non-transfected cell. These results showed an increase in the percentage of phagocytosis and PI in ANXA1 knockdown cells.

Removal of apoptotic cells by phagocytosis is a crucial process in inflammation prevention. Signals displayed by apoptotic cells, such as 'find-me' and 'eat-me' signals, draw the attention of a macrophage to phagocyte them (24). The results showed the efficiency of macrophage in removing damaged or apoptotic cells were increased after ANXA1 knockdown. Knockdown of ANXA1 induced apoptosis and caused apoptotic cells to display 
'eat-me' signals such as phosphatidylserine (PtdSer), fractalkine, sphingosine-1-phosphate (S1P) and lysophosphatidylcholine (LPC); which are released from apoptotic and necrotic cells to attract phagocytes to the apoptotic cell (25). Therefore, signals produced by both apoptotic and necrotic cells attract macrophages to engulf them.

\section{CONCLUSIONS}

This finding concludes that ANXA1 is an important protein in human leukemia cells and knockdown of ANXA1 presents the potential treatment strategy due to the ability to induce apoptosis, cause cell cycle arrest and phagocytosis in leukemia cells. Besides, the results indicated that ANXA1 functions as an anti-apoptotic protein and ANXA1 knockdown disrupts normal conditions in cancer cells causing cell cycle arrest and apoptosis. The unique characteristic of ANXA1 knockdown in inducing apoptosis through the different mechanisms for each cell line convinces that this protein exhibits different effects based on cell-type behavior; an intrinsic pathway for U937 cell and an extrinsic pathway for Jurkat and K562 cells that contribute more information about this protein. These findings can contribute to further understanding of ANXA1 role in human leukemic cell lines.

Acknowledgement. - This project has been funded by the Ministry of Higher Education for Fundamental Research Grant Scheme (FRGS), FRGS/1/2015/SKK09/UKM/02/3. The authors acknowledge and thank the Faculty of Health Science, UKM for the BD FacsCanto II flow cytometry.

\section{REFERENCES}

1. R. Belvedere, V. Bizzarro, A. Popolo, F. Dal Piaz, M. Vasaturo, P. Picardi, L. Parente and A. Petrella, Role of intracellular and extracellular annexin A1 in migration and invasion of human pancreatic carcinoma cells, BMC cancer 14 (2014) 961-975; https://doi.org/10.1186/1471-2407-14-961

2. A. Sabran, E. Kumolosasi and I. Jantan, Effects of annexin A1 on apoptosis and cell cycle arrest in human leukemic cell lines, Acta Pharm. 69 (2019) 75-86; http://doi.org/10.2478/acph-2019-0005

3. E. Solito, C. De Coupade, S. Canaider, N. J. Goulding and M. Perretti, Transfection of annexin 1 in monocytic cells produces a high degree of spontaneous and stimulated apoptosis associated with caspase-3 activation, Br. J. Pharmacol. 133 (2001) 217-228; https://doi.org/10.1038/sj.bjp.0704054

4. C. W. D'Acunto, B. Fontanella, M. Rodriquez, M. Taddei, L. Parente and A. Petrella, Histone deacetylase inhibitor FR235222 sensitizes human prostate adenocarcinoma cells to apoptosis through up-regulation of Annexin A1, Cancer Lett. 295 (2010) 85-91; https://doi.org/10.1016/j.canlet.2010.02.016

5. R. Debret, H. El Btaouri, L. Duca, I. Rahman, S. Radke, B. Haye, J. M. Sallenave and F. Antonicelli, Annexin A1 processing is associated with caspase-dependent apoptosis in BZR cells, FEBS Lett. 546 (2003) 195-202; https://doi.org/10.1016/s0014-5793(03)00570-2

6. Y. Feng, J. Wu, X. Feng, D. Tao, J. Hu, J. Qin, X. Li, W. Xiao, K. Gardner, S. I. V. Judge, Q. Q. Li and $\mathrm{J}$. Gong, Timing of apoptosis onset depends on cell cycle progression in peripheral blood lymphocytes and lymphocytic leukemia cells, Oncol. Rep. 17 (2007) 1437-1444; https://doi.org/10.3892/ or.17.6.1437

7. S. Fulda and K. M. Debatin, Extrinsic versus intrinsic apoptosis pathways in anticancer chemotherapy, Oncogene 25 (2006) 4798-4811; https://doi.org/10.1038/sj.onc.1209608 
8. S. Inoue, G. Browne, G. Melino and G. M. Cohen, Ordering of caspases in cells undergoing apoptosis by the intrinsic pathway, Cell Death Differ. 16 (2009) 1053-1061; https://doi.org/10.1038/ cdd.2009.29

9. E. Tothova, M. Fricova, N. Stecova, A. Kafkova and A. Elbertova, High expression of Bcl-2 protein in acute myeloid leukemia cells is associated with poor response to chemotherapy, Neoplasma 49 (2002) 141-144; https://pubmed.ncbi.nlm.nih.gov/12097997

10. A. Petrella, C. W. D'Acunto, M. Rodriquez, M. Festa, A. Tosco, I. Bruno, S. Terracciano, M. Taddei, L. G. Paloma and L. Parente, Effects of FR235222, a novel HDAC inhibitor, in proliferation and apoptosis of human leukaemia cell lines: role of annexin A1, Eur. J. Cancer 44 (2008) 740-749; https://doi.org/10.1016/j.ejca.2008.01.023

11. D. M. Patel, S. F. Ahmad, D. G. Weiss, V. Gerke and S. A. Kuznetsov, Annexin A1 is a new functional linker between actin filaments and phagosomes during phagocytosis, J. Cell Sci. 124 (2011) 578-588; https://doi.org/10.1242/jcs.076208

12. Z. Chen, E. Yoshihara, A. Son, Y. Matsuo, H. Masutani, K. Sugie, M. Maeda and J. Yodoi, Differential roles of Annexin A1 (ANXA1/lipocortin-1/lipomodulin) and thioredoxin binding protein-2 (TBP-2/VDUP1/TXNIP) in glucocorticoid signaling of HTLV-I-transformed T cells, Immunol. Lett. 13 (2010) 11-18; https://doi.org/10.1016/j.imlet.2010.04.003

13. Y. L. Wu, X. R. Jiang, D. M. Lillington, A. C. Newland and S. M. Kelsey, Upregulation of lipocortin 1 inhibits tumour necrosis factor-induced apoptosis in human leukaemic cells: a possible mechanism of resistance to immune surveillance, Br. J. Haematol. 111 (2000) 807-816; https://onlinelibrary. wiley.com/doi/pdf/10.1111/j.1365-2141.2000.02397.x

14. C. Jia, D. Kong, Y. Guo, L. Li and L. Quan, Enhanced antitumor effect of combination of annexin A1 knockdown and bortezomib treatment in multiple myeloma in vitro and in vivo, Biochem. Biophis. Res. Commun. 505 (2018) 720-725; https://doi.org/10.1016/j.bbrc.2018.09.140

15. E. Uribe-Querol and C. Rosales, Control of phagocytosis by microbial pathogens, Front. Immunol. 8 (2017) 1-23; https://doi.org/10.3389/fimmu.2017.01368

16. J. Feng, X. Wang, H. Li, L. Wang and Z. Tang, Silencing of Annexin A1 suppressed the apoptosis and inflammatory response of preeclampsia rat trophoblasts, Int. J. Mol. Med. 42 (2018) 3125-3134; https://doi.org/10.3892/ijmm.2018.3887

17. D. R. Green, Apoptotic pathways: Ten minutes to dead, Cell. 121 (2005) 671-674; https://doi.org/ 10.1016/j.cell.2005.05.019

18. V. D. Moore and A. Letai, Rational design of therapeutics targeting the BCL-2 family: are some cancer cells primed for death but waiting for a final push? Adv. Exp. Med. Biol. 615 (2008) 159-175; https://doi.org/10.1007/978-1-4020-6554-5_8

19. L. Franchi, T. Eigenbrod, R. Muñoz-Planillo and G. Nuñez, The inflammasome: a caspase-1-activation platform that regulates immune responses and disease pathogenesis, Nature Immunol. 10 (2009) 241-247; https://www.nature.com/articles/ni.1703

20. N. Vergnolle, P. Pagès, R. Guimbaud, S. Chaussade, L. Buéno, J. Escourrou and C. Comera, Annexin 1 is secreted in situ during ulcerative colitis in humans, Inflamm. Bowel Dis. 10 (2004) 584592; https://doi.org/10.1097/00054725-200409000-00013

21. A. Denes, G. Lopez-Castejon and D. Brough, Caspase-1: is IL-1 just the tip of the ICEberg? Cell Death Dis. 3 (2012) e338-e346; https://doi.org/10.1038/cddis.2012.86

22. Q. H. Liu, M. L. Shi, J. Bai and J. N. Zheng, Identification of ANXA1 as a lymphatic metastasis and poor prognostic factor in pancreatic ductal adenocarcinoma, Asian Pac. J. Cancer Prev. 16 (2015) 2719-2724; https://doi.org/10.7314/apjcp.2015.16.7.2719

23. G. Li, S. He, L. Chang, H. Lu, H. Zhang, H. Zhang and J. Chiu, GADD45 $\alpha$ and annexin A1 are involved in the apoptosis of HL-60 induced by resveratrol, Phytomedicine 18 (2011) 704-709; https:// doi.org/10.1016/j.phymed.2010.11.015 
M. Hasan et al:: Knockdown of Annexin A1 induces apoptosis, causing G2/M arrest and facilitating phagocytosis activity in human leukemia cell lines, Acta Pharm. 72 (2022) 109-122.

24. S. Y. Park and I. S. Kim, Engulfment signals and the phagocytic machinery for apoptotic cell clearance, Exp. Mol. Med. 49 (2017) e331-e340; https://doi.org/10.1038/emm.2017.52

25. I. K. H. Poon, C. D. Lucas, A. G. Rossi and K. S. Ravichandran, Apoptotic cell clearance: basic biology and therapeutic potential, Nat. Rev. Immunol. 14 (2014) 166-180; https://doi.org/10.1038/nri3607 